\title{
Pyramidal Tract Syndrome, CTCAE
}

National Cancer Institute

\section{Source}

National Cancer Institute. Pyramidal Tract Syndrome, CT CAE. NCI Thesaurus. Code C143795.

A disorder characterized by dysfunction of the corticospinal (pyramidal) tracts of the spinal cord. Symptoms include an increase in the muscle tone in the lower extremities, hyperreflexia, positive Babinski and a decrease in fine motor coordination. 